\title{
Comparative analysis of the results from static strength calculations and strength tests of an Y25Ls-K bogie frame
}

\author{
Svetoslav Slavchev ${ }^{1, *}$, Vladislav Maznichki ${ }^{1}$, Valeri Stoilov ${ }^{1}$, and Sanel Purgic ${ }^{1}$ \\ ${ }^{1}$ Technical University - Sofia, "Railway engineering“ Department, 8 Kliment Ohridski Blvd., 1172 Sofia, Bulgaria
}

\begin{abstract}
The comparative analysis is based on the results of the static strength analysis of an Y25Ls-K bogie frame and the tests that have been carried out. Strength calculations have been made using the Finite Elements Method at the Department of Railway Engineering at the Technical University - Sofia and the tests of the bogie frame have been conducted in VUZ Testing laboratory in the Czech Republic. It was found out that the stress results are very similar, especially in the areas with maximal values. This proves that a suitable calculation model with a relatively small number of finite elements can be developed. This allows us to solve a wide range of problems concerning the improvement of the bogie frame with similar construction.
\end{abstract}

\section{Introduction}

This paper presents a comparative analysis of the static strength analysis and tests conducted on supporting steel structure of an Y25Ls-K bogie frame.

Strength calculations have been made using the Finite Elements Method [1,2] at the Department of Railway Engineering of the Technical University Sofia. The tests of the Y25Ls-K bogie frame have been carried out in the Výzkumný Ústav Železniční a.s.- VUZ Testing laboratory in the Czech Republic [3].

Theoretical and experimental studies have been done in full compliance with European standard EN 13749:2011 [4], EN 12663-2:2010 [5], TSI "Rolling stock" [6], and Leaflets 510-3 and 615-4 [7,8] of the International Railway Union (UIC). According to those documents 13 load cases for study of stresses and displacements in the bogie frame construction are provided. Due to the limited size of the paper it presents only the results of five load cases marked in Table 1 with S1-S5. It should be noted that the number of points where the comparison is carried out is also limited. Only those that have relatively high stress values or are located in zones with section changes were studied.

\section{Loading methodology}

In accordance with the international requirements, each newly-built bogie is subjected to theoretical and native studies to demonstrate the strength of its structure. Types and conditions of loading are regulated by the European Standard EN 13749:2011 [4], the TSI [6] and the Leaflets of International Railways Union (UIC) 510-3 and 615-4 [7, 8]. According to the documents cited, the size and direction of the forces acting on the bogie depend on the load case (Fig. 1).

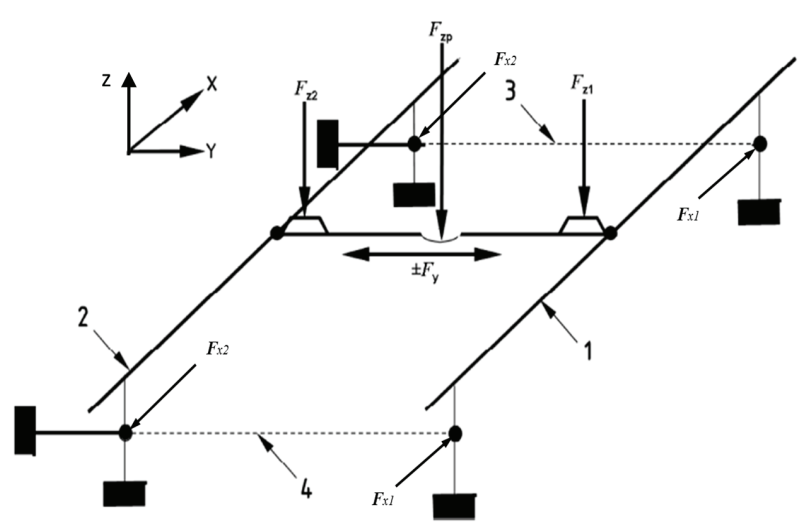

Fig. 1. Forces acting on bogie frame according to [4].

The forces acting on the bogie frame are defined in equations given in $[4,10]$, as follows:

- Vertical forces

$$
F_{z}=4 Q_{0}-m^{+} g
$$

In the case when the force is applied only to the pivot:

$$
F_{z c}=2 F_{z} .
$$

For cases when the force is applied to both the pivot and one sidebearer:

$$
\begin{gathered}
F_{z c 1}=1,5 F_{z}(1-\alpha) ; \\
F_{z c 2}=1,5 F_{z} \alpha .
\end{gathered}
$$

where: $F_{z}$ - total vertical load supported by the bogie; $Q_{0}$ - static vertical force at the level of the wheel for a loaded wagon, for axle load $p_{0}=22,5$ t: $Q_{0}=0,5 p_{0} g=$ $110 \mathrm{kN} ; m^{+}-$bogie mass $(4128 \mathrm{~kg}) ; g$ - acceleration due to gravity; $F_{z c}$ - force applied only to the pivot;

* Corresponding author: slavchev_s_s@tu-sofia.bg 
$F_{z 1}$ and $F_{z 2}$ - forces applied to the sidebearers. For UIC service with a distance between the sidebearers of $1,7 \mathrm{~m}$, $\alpha$ is taken as $0,3 . F_{b z}$ - vertical brake force defined in accordance to [10].

- Transverse force

$$
F_{y}=20+\left(F_{z}+m^{+} g\right) / 3 .
$$

- Longitudinal lozenging forces

$$
F_{x}=0,1\left(F_{z}+m^{+} g\right) .
$$

$F_{b x}$ - longitudinal brake force defined in accordance with [10]. Brake force depends on the type of frame structure (axle box guidance, type of brake rigging etc. [10]).

- Twist loading $g^{+}-$the bogie frame should withstand the loads resulting from a track twist of $10 \%$.

Summing up the results of the equations given above, the following load cases combining the calculated forces (in $\mathrm{kN}$ ) are obtained (Table 1).

Table 1. Applied load cases. All forces in $[\mathrm{kN}]$.

\begin{tabular}{|l|c|c|c|c|c|c|c|c|}
\hline & $F_{z 2}$ & $F_{z c}$ & $F_{z 1}$ & $F_{y}$ & $g+$ & $F_{x}$ & $F_{b x}$ & $F_{b z}$ \\
\hline S1 & & 801,9 & & & & & & \\
\hline S2 & & 421,0 & 180,4 & & $+10 \%$ & & & \\
\hline S3 & & 421,0 & 180,4 & & $-10 \%$ & & & \\
\hline S4 & 180,4 & 421,0 & & & $+10 \%$ & & & \\
\hline S5 & 180,4 & 421,0 & & & $-10 \%$ & & & \\
\hline S6 & & 421,0 & 180,4 & 167,2 & & & & \\
\hline S7 & 180,4 & 421,0 & & $-167,2$ & & & & \\
\hline S8 & & 601,4 & & & & 44,1 & & \\
\hline S9 & & 601,4 & & & & $-44,1$ & & \\
\hline S10 & & 400,9 & & & & 202,6 & & \\
\hline S1 & & 400,9 & & & & $-202,6$ & & \\
\hline S12 & & 481,1 & & & & & 98,8 & 24,7 \\
\hline S13 & & 481,1 & & & & & $-98,8$ & $-24,7$ \\
\hline
\end{tabular}

\section{Calculation models}

From the analysis of the design documentation we have determined the bearing elements of the structure, the materials from which it is built and the technology of manufacturing (type of welds, additional machining, etc.). On the basis of this, the calculation models were designed [9] as shown in Fig. 2.

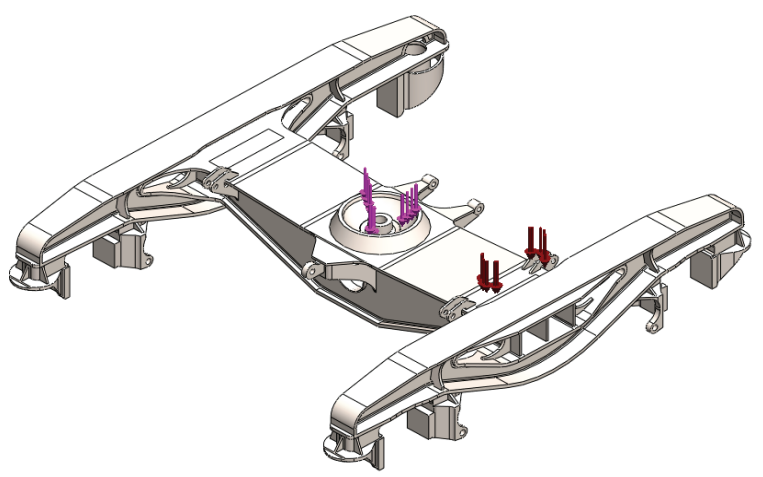

Fig. 2. Calculation model.
In this model the finite elements mesh is compressed (1 374520 nodes and 843616 elements), the maximum size of finite elements is $15 \mathrm{~mm}$ (Fig. 3), which shows a very good density of the analysed quantities.

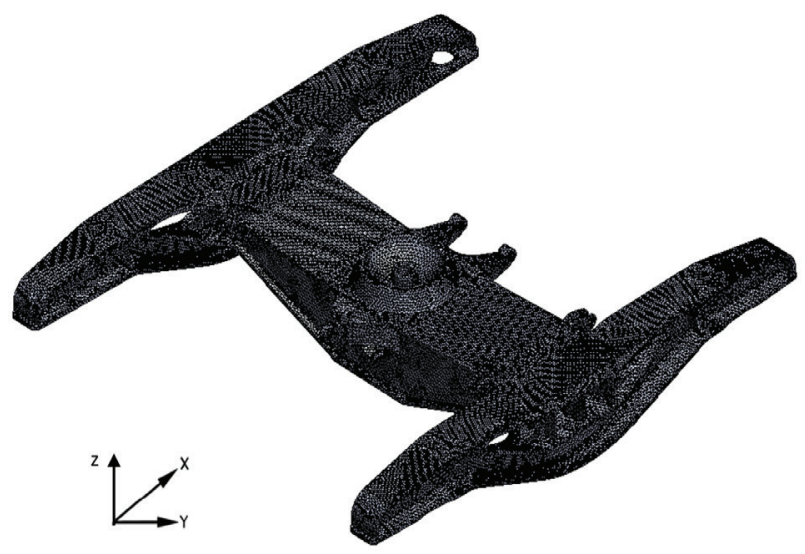

Fig. 3. Finite elements mesh of calculation model.

\section{Results analysis}

During the tests, stresses in terms of value and distribution, similar to those obtained theoretically, have been registered. A comparative analysis of the results is presented in Table 2 where the following indications are used: the strain gauge number/number of its corresponding element in the calculation model (Fig. 4), the test results in MPa are presented in the same row; $\sigma_{x}$, $\sigma_{y}$, and $\sigma_{z}$ are the components of the stress tensor (7) on the main inertia axes $x, y, z$ in MPa:

$$
\sigma_{i j}=\left[\begin{array}{ccc}
\sigma_{x} & \tau_{y x} & \tau_{z x} \\
\tau_{x y} & \sigma_{y} & \tau_{z y} \\
\tau_{x z} & \tau_{y z} & \sigma_{z}
\end{array}\right] ;
$$

von Mises stress results from FEM-calculation, von Mises stresses calculated with equation (8);

$$
\sigma_{v M}=\sqrt{\frac{\left(\sigma_{x}-\sigma_{y}\right)^{2}+\left(\sigma_{y}-\sigma_{z}\right)^{2}+\left(\sigma_{z}-\sigma_{x}\right)^{2}+6\left(\tau_{x y}^{2}+\tau_{y z}^{2}+\tau_{z x}^{2}\right)}{2}}
$$

S1- S5 are codes of analyzed load cases.

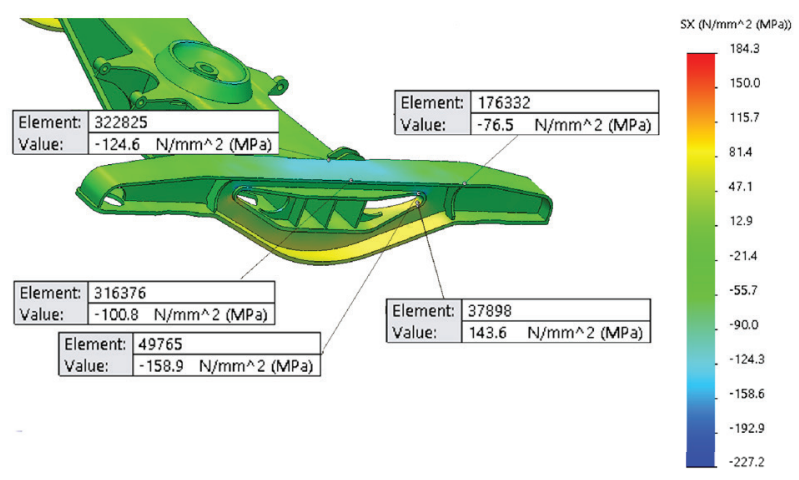

Fig. 4. Stress values obtained in elements corresponding to strain gauges 
Table 2. Compared stress values.

\begin{tabular}{|c|c|c|c|c|c|c|c|}
\hline DMS/Element & S1 & S2 & S3 & S4 & S5 & $\max$ & $\min$ \\
\hline T111/316376 & -85 & -47 & -51 & -70 & -75 & -47 & -85 \\
\hline$\sigma_{x}$ & $-100,8$ & $-61,4$ & $-66,5$ & -87 & $-92,1$ & -61 & -101 \\
\hline$\sigma_{y}$ & $-0,1$ & 0,1 & 0,1 & 0 & 0,1 & 0 & 0 \\
\hline$\sigma_{z}$ & 0 & 0 & 0 & $-0,1$ & $-0,1$ & $\mathbf{0}$ & $\mathbf{0}$ \\
\hline von Mises & 100,8 & 92,6 & 66,5 & 87 & 92,1 & 101 & 67 \\
\hline T112/322825 & -119 & -68 & -65 & -126 & -119 & -65 & -126 \\
\hline$\sigma_{x}$ & $-124,6$ & $-63,5$ & $-57,5$ & $-121,2$ & $-115,2$ & -58 & -125 \\
\hline$\sigma_{y}$ & 0 & $-0,8$ & $-0,7$ & $-1,4$ & $-1,3$ & 0 & -1 \\
\hline$\sigma_{z}$ & 0 & $-0,1$ & $-0,1$ & $-0,1$ & $-0,1$ & $\mathbf{0}$ & $\mathbf{0}$ \\
\hline von Mises & 124,6 & 113 & 57,1 & 120,4 & 114,5 & 125 & 57 \\
\hline T312/314726 & -112 & -123 & -131 & -64 & -66 & -64 & -131 \\
\hline$\sigma_{x}$ & $-124,2$ & $-121,2$ & $-128,2$ & $-59,6$ & $-66,6$ & -60 & -128 \\
\hline$\sigma_{y}$ & 0,1 & $-1,7$ & $-1,7$ & $-0,9$ & $-0,9$ & 0 & -2 \\
\hline$\sigma_{z}$ & 0 & $-0,2$ & $-0,2$ & $-0,1$ & $-0,1$ & 0 & 0 \\
\hline von Mises & 124,3 & 65,9 & 127,4 & 59,1 & 66,2 & 127 & 59 \\
\hline T213/176332 & -76 & -39 & -24 & -70 & -58 & -24 & -76 \\
\hline$\sigma_{x}$ & $-76,5$ & $-57,9$ & $-36,9$ & $-76,4$ & $-55,4$ & -37 & -77 \\
\hline$\sigma_{y}$ & $-0,8$ & $-0,7$ & $-0,3$ & -1 & $-0,6$ & $\mathbf{0}$ & -1 \\
\hline$\sigma_{z}$ & 0 & $-0,1$ & 0 & 0 & 0,1 & 0 & $\mathbf{0}$ \\
\hline von Mises & 76,1 & 73,7 & 36,7 & 76 & 55,1 & 76 & 37 \\
\hline $\mathrm{T} 214 / 157611$ & -85 & -76 & -56 & -91 & -76 & -56 & -91 \\
\hline$\sigma_{x}$ & $-59,4$ & -42 & $-14,5$ & $-74,6$ & -47 & -15 & -75 \\
\hline$\sigma_{y}$ & $-0,5$ & $-0,7$ & $-0,3$ & -1 & $-0,7$ & $\mathbf{0}$ & -1 \\
\hline$\sigma_{z}$ & 0 & 0,1 & 0 & 0,1 & 0 & $\mathbf{0}$ & $\mathbf{0}$ \\
\hline von Mises & 59,1 & 65,4 & 14,4 & 74,1 & 46,7 & 74 & 14 \\
\hline T315/305644 & -84 & -77 & -56 & -37 & -48 & -37 & -84 \\
\hline$\sigma_{x}$ & $-74,2$ & $-61,9$ & $-69,6$ & -45 & $-52,8$ & -45 & -74 \\
\hline$\sigma_{y}$ & $-2,4$ & -2 & $-2,1$ & $-1,7$ & $-1,8$ & -2 & -2 \\
\hline$\sigma_{z}$ & 0,2 & 0,5 & 1 & 0,1 & 0,6 & 1 & 0 \\
\hline von Mises & 76,4 & 43,5 & 72,2 & 46,5 & 54,7 & 76 & 44 \\
\hline T316/309615 & 70 & 59 & 89 & 33 & 45 & 89 & 33 \\
\hline$\sigma_{x}$ & 73,1 & 65,4 & 81,4 & 30,4 & 46,4 & 81 & 30 \\
\hline$\sigma_{y}$ & 0 & 0 & $-0,1$ & 0 & $-0,1$ & $\mathbf{0}$ & $\mathbf{0}$ \\
\hline$\sigma_{z}$ & 17,5 & 16,1 & 22,1 & 6,8 & 12,8 & 22 & 7 \\
\hline von Mises & 88,8 & 49,4 & 102 & 36,6 & 58,4 & 102 & 37 \\
\hline T119/246268 & 87 & 49 & 51 & 82 & 84 & 87 & 49 \\
\hline$\sigma_{x}$ & 119,4 & 64,4 & 64,3 & 108,7 & 108,7 & 119 & 64 \\
\hline$\sigma_{y}$ & 84,1 & 44,3 & 44,6 & 73,7 & 74,1 & 84 & 44 \\
\hline$\sigma_{z}$ & -1 & -1 & -1 & $-1,5$ & $-1,5$ & -1 & -2 \\
\hline von Mises & 107,2 & 97,1 & 58 & 97,6 & 97,6 & 107 & 58 \\
\hline T319/405348 & 92 & 87 & 88 & 52 & 52 & 92 & 52 \\
\hline$\sigma_{x}$ & 119,4 & 109,8 & 109,2 & 64,4 & 63,9 & 119 & 64 \\
\hline$\sigma_{y}$ & 83 & 72 & 70,7 & 42,2 & 41 & 83 & 41 \\
\hline$\sigma_{z}$ & $-0,1$ & 0,1 & 0 & 0,1 & 0 & 0 & 0 \\
\hline von Mises & 106,1 & 55,9 & 95,9 & 56,7 & 56,1 & 106 & 56 \\
\hline T322/238708 & 58 & 47 & 33 & 34 & 41 & 58 & 33 \\
\hline$\sigma_{x}$ & 27,9 & 47,6 & 42 & 0,7 & -5 & 48 & -5 \\
\hline$\overline{\sigma_{y}}$ & 0,5 & 0,5 & 0,7 & 0,5 & 0,6 & 1 & 1 \\
\hline$\sigma_{z}$ & 1,1 & 2,2 & 1,8 & $-0,1$ & $-0,4$ & 2 & 0 \\
\hline von Mises & 28,9 & 17,2 & 43,5 & 1,8 & 5,7 & 44 & 2 \\
\hline T32/467786 & -117 & -102 & -163 & -53 & -83 & -53 & -163 \\
\hline$\sigma_{x}$ & $-130,5$ & $-111,7$ & $-147,2$ & $-48,7$ & $-84,2$ & -49 & -147 \\
\hline$\sigma_{y}$ & $-0,6$ & $-0,7$ & -1 & $-0,3$ & $-0,6$ & 0 & -1 \\
\hline$\sigma_{z}$ & 0,2 & 0 & $-0,1$ & 0 & $-0,1$ & $\mathbf{0}$ & 0 \\
\hline von Mises & 130,7 & 73 & 147,2 & 48,6 & 84,2 & 147 & 49 \\
\hline T325/263874 & 84 & 81 & 76 & 32 & 55 & 84 & 32 \\
\hline$\sigma_{x}$ & 65,7 & 54,9 & 59,4 & 36,4 & 40,9 & 66 & 36 \\
\hline$\sigma_{y}$ & 1,2 & 1,1 & 1,2 & 0,7 & 0,8 & 1 & 1 \\
\hline$\sigma_{z}$ & 12,9 & 11,4 & 12,8 & 7,3 & 8,8 & 13 & 7 \\
\hline von Mises & 79 & 50,7 & 72,8 & 43,9 & 50,1 & 79 & 44 \\
\hline T229/198779 & -89 & -48 & -57 & -57 & -69 & -48 & -89 \\
\hline$\sigma_{x}$ & $-23,2$ & $-11,6$ & $-14,4$ & $-12,4$ & $-15,2$ & -12 & -23 \\
\hline$\sigma_{y}$ & $-53,9$ & -29 & $-31,5$ & $-30,5$ & -33 & -29 & -54 \\
\hline$\sigma_{z}$ & 0,1 & 0 & 0 & 0,1 & 0 & $\mathbf{0}$ & 0 \\
\hline von Mises & 75,5 & 46,3 & 44,8 & 42 & 47 & 76 & 42 \\
\hline T330/379240 & 82 & 52 & 41 & 43 & 47 & 82 & 41 \\
\hline
\end{tabular}

\begin{tabular}{|c|c|c|c|c|c|c|c|}
\hline$\sigma_{x}$ & $-1,5$ & $-1,3$ & $-1,5$ & $-1,3$ & $-1,5$ & $\mathbf{- 1}$ & $\mathbf{- 2}$ \\
\hline$\sigma_{y}$ & 61,5 & 37,2 & 37,9 & 35,9 & 36,5 & $\mathbf{6 2}$ & $\mathbf{3 6}$ \\
\hline$\sigma_{z}$ & 0,7 & 0,3 & 0,4 & 0,3 & 0,4 & $\mathbf{1}$ & $\mathbf{0}$ \\
\hline von Mises & 64,4 & 38,3 & 39,7 & 37,4 & 38,3 & $\mathbf{6 4}$ & $\mathbf{3 7}$ \\
\hline T430/377102 & 82 & 41 & 51 & 45 & 42 & $\mathbf{8 2}$ & $\mathbf{4 1}$ \\
\hline$\sigma_{x}$ & -2 & $-1,7$ & $-1,6$ & $-1,7$ & $-1,6$ & $\mathbf{- 2}$ & $\mathbf{- 2}$ \\
\hline$\sigma_{y}$ & 58 & 37,5 & 36,6 & 36,5 & 35,6 & $\mathbf{5 8}$ & $\mathbf{3 6}$ \\
\hline$\sigma_{z}$ & 0,8 & 0,5 & 0,4 & 0,4 & 0,4 & $\mathbf{1}$ & $\mathbf{0}$ \\
\hline von Mises & 61,1 & 38,7 & 38,4 & 38,4 & 37,3 & $\mathbf{6 1}$ & $\mathbf{3 7}$ \\
\hline T331/188444 & 108 & 21 & 218 & 18 & 123 & $\mathbf{2 1 8}$ & $\mathbf{1 8}$ \\
\hline$\sigma_{x}$ & 12,8 & 8,2 & 14,3 & 4,3 & 10,3 & $\mathbf{1 4}$ & $\mathbf{4}$ \\
\hline$\sigma_{y}$ & 129,7 & 91,5 & 141,3 & 47 & 96,8 & $\mathbf{1 4 1}$ & $\mathbf{4 7}$ \\
\hline$\sigma_{z}$ & 12,7 & 8 & 13,6 & 4,5 & 10,1 & $\mathbf{1 4}$ & $\mathbf{5}$ \\
\hline von Mises & 137,7 & 104 & 150,4 & 49,7 & 104,1 & $\mathbf{1 5 0}$ & $\mathbf{5 0}$ \\
\hline T431/297427 & 128 & 206 & 2 & 112 & 5 & $\mathbf{2 0 6}$ & $\mathbf{2}$ \\
\hline$\sigma_{x}$ & 13,6 & 16,3 & 9,4 & 11,4 & 4,6 & $\mathbf{1 6}$ & $\mathbf{5}$ \\
\hline$\sigma_{y}$ & 132,6 & 146,1 & 95,9 & 97,9 & 47,7 & $\mathbf{1 4 6}$ & $\mathbf{4 8}$ \\
\hline$\sigma_{z}$ & 13,9 & 15,5 & 9,5 & 11 & 4,9 & $\mathbf{1 6}$ & $\mathbf{5}$ \\
\hline von Mises & 141 & 49,1 & 100,3 & 105 & 50,1 & $\mathbf{1 4 1}$ & $\mathbf{4 9}$ \\
\hline T132/84072 & -136 & -67 & -87 & -118 & -150 & $\mathbf{- 6 7}$ & $\mathbf{- 1 5 0}$ \\
\hline$\sigma_{x}$ & $-143,3$ & -77 & $-78,4$ & -131 & $-132,4$ & $\mathbf{- 7 7}$ & $\mathbf{- 1 4 3}$ \\
\hline$\sigma_{y}$ & 3,9 & $-0,2$ & 2,1 & $-1,4$ & 1 & $\mathbf{4}$ & $\mathbf{- 1}$ \\
\hline$\sigma_{z}$ & $-69,9$ & $-39,1$ & $-40,3$ & $-65,8$ & -67 & $\mathbf{- 3 9}$ & $\mathbf{- 7 0}$ \\
\hline von Mises & 195 & 179,7 & 106,8 & 175,5 & 179,9 & $\mathbf{1 9 5}$ & $\mathbf{1 0 7}$ \\
\hline T232/49765 & -172 & -111 & -77 & -174 & -137 & $\mathbf{- 7 7}$ & $\mathbf{- 1 7 4}$ \\
\hline$\sigma_{x}$ & $-158,9$ & $-81,2$ & $-82,2$ & $-136,3$ & $-137,4$ & $\mathbf{- 8 1}$ & $\mathbf{- 1 5 9}$ \\
\hline$\sigma_{y}$ & 5,6 & 3,5 & $-1,3$ & 6,1 & 1,3 & $\mathbf{6}$ & $\mathbf{- 1}$ \\
\hline$\sigma_{z}$ & $-70,8$ & $-35,2$ & -33 & $-58,8$ & $-56,7$ & $\mathbf{- 3 3}$ & $\mathbf{- 7 1}$ \\
\hline von Mises & 206,2 & 148,7 & 100,5 & 178,4 & 172,4 & $\mathbf{2 0 6}$ & $\mathbf{1 0 1}$ \\
\hline T237/37898 & 155 & 95 & 68 & 161 & 124 & $\mathbf{1 6 1}$ & $\mathbf{6 8}$ \\
\hline$\sigma_{x}$ & 143,6 & 77,8 & 80,4 & 136,6 & 139,2 & $\mathbf{1 4 4}$ & $\mathbf{7 8}$ \\
\hline$\sigma_{y}$ & $-12,3$ & $-2,8$ & $-5,3$ & $-5,8$ & $-8,4$ & $\mathbf{- 3}$ & $\mathbf{- 1 2}$ \\
\hline$\sigma_{z}$ & 69,3 & 37,5 & 40,5 & 66,7 & 69,7 & $\mathbf{7 0}$ & $\mathbf{3 8}$ \\
\hline von Mises & 204,6 & 162,9 & 114,7 & 191,1 & 197,6 & $\mathbf{2 0 5}$ & $\mathbf{1 1 5}$ \\
\hline & & & & & & & \\
\hline
\end{tabular}

The analysis of the data in Table 2 shows that there is a very good match of the results. Differences in stress values can be explained by the simplifications adopted in the modeling: the way of gluing and positioning of the strain gauges; measuring errors of equipment; inaccuracies in the manufacturing of the bearing structure; technological deviations of the material; the presence of serious stress notches etc.

Table 3 presents data for the forces applied in five load cases considered in the paper (Figures 5 and 6). In vertical direction, very good matching can be observed and the test forces differ slightly from the calculated ones. This can be explained by the errors of the measuring equipment and the asymmetry of the structure.

Table 3. Applied vertical forces in $\mathrm{kN}$.

\begin{tabular}{|c|c|c|c|c|c|}
\hline & $\mathrm{S} 1$ & $\mathrm{~S} 2$ & $\mathrm{~S} 3$ & $\mathrm{~S} 4$ & $\mathrm{~S} 5$ \\
\hline$F_{z 1}$ & $-399,0$ & $-223,5$ & $-223,9$ & $-377,0$ & $-376,8$ \\
\hline$F_{z 2}$ & $-399,9$ & $-376,3$ & $-376,8$ & $-222,4$ & $-224,1$ \\
\hline sum $F_{z}$ & $-799,0$ & $-599,8$ & $-600,7$ & $-599,4$ & $-600,9$ \\
\hline$F_{z}$ FEM & 801,9 & 601,4 & 601,4 & 601,4 & 601,4 \\
\hline
\end{tabular}

Table 4 shows the reaction data $R_{z 1}-R_{z 4}$ in bearings in the vertical direction (Figures 5 and 6) for the asymmetrical load cases S2 to S5. A very good match can be seen, excluding the values of the bearing reaction, where the track twist is imitated. 


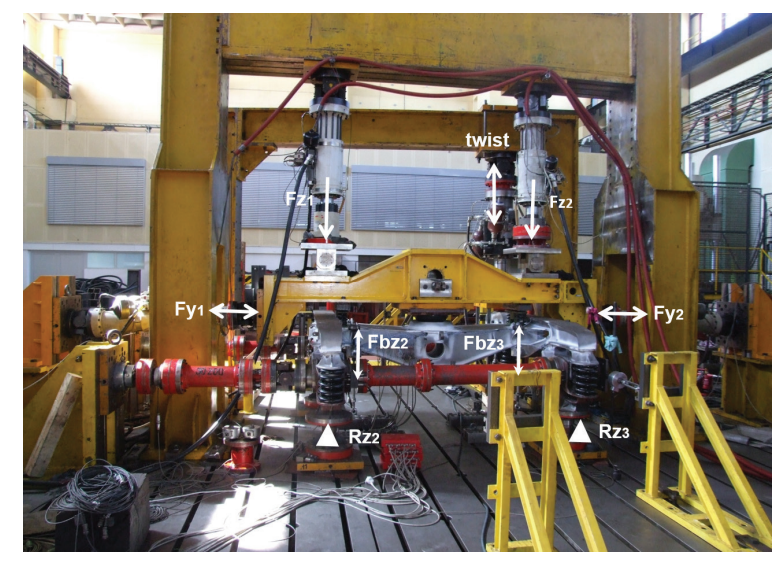

Fig. 5. Test rig at Výzkumný Ústav Železniční a.s. (VUZ) [3].

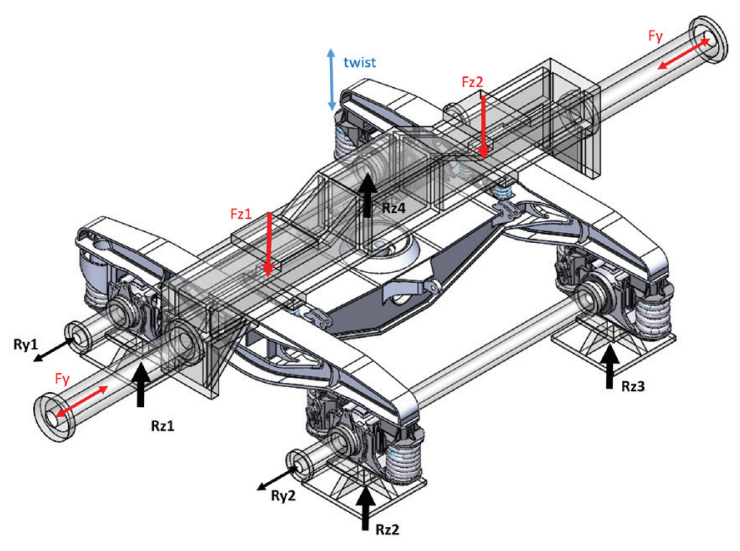

Fig. 6. Bearing reactions.

Table 4. Bearing reactions in $[\mathrm{kN}]$.

\begin{tabular}{|c|c|c|c|c|}
\hline & $R_{z 1}$ & $R_{z 2}$ & $R_{z 3}$ & $R_{z 4}$ \\
\hline \multirow{2}{*}{ S2 } & 88,2 & 134,6 & 146,1 & 212,8 \\
\cline { 2 - 5 } & 101,5 & 123,2 & 172,0 & 224,3 \\
\hline \multirow{2}{*}{ S3 } & 133,9 & 88,5 & 212,8 & 146,8 \\
\cline { 2 - 5 } & 123,2 & 101,8 & 204,7 & 151,7 \\
\hline \multirow{2}{*}{ S4 } & 179,8 & 200,9 & 83,5 & 136,8 \\
\cline { 2 - 5 } & 177,4 & 199,2 & 96,2 & 148,6 \\
\hline \multirow{2}{*}{ S5 } & 212,8 & 151,7 & 133,8 & 88,3 \\
\cline { 2 - 5 } & 199,0 & 177,8 & 128,9 & 76,0 \\
\hline
\end{tabular}

This can be explained by a difference in the position where the frame displacement (as a consequence of the track twist) is applied. If the force is applied under the support of the measuring equipment, then the magnitude of the reaction would increase. Similarly, if the force is applied above the support, the magnitude of the reaction would decrease with the value of the force produced by the track twisting (Fig. 7).

\section{Conclusions and recommendations}

when summarizing the overall work done on this study the following conclusions and recommendations can be made:

1. Sophisticated calculation models for the strength analysis of the Y25Ls-K bogie frame have been developed, consisting of 843616 finite elements that accurately describe the geometry of the object.

2 . In the process of strength testing minor design changes of the Y25Ls-K bogie frame were made.

3 . The analysis of the results in Tables 2 and 3 shows that the stress distribution obtained theoretically is very close to the stress distribution recorded in the test.

4. The relative error of stress results is relatively small, which allows for developed calculation models to be used in order to study other similar bogie frame designs as well as to optimize the tested bogie.

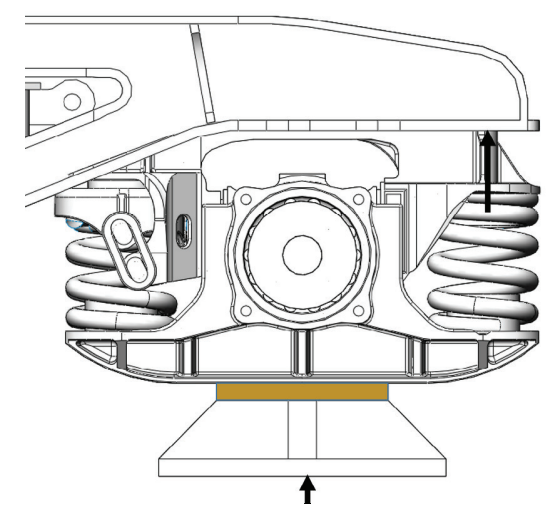

Fig. 7. Reaction under the support.

\section{References}

1. O. Zenkiewicz, The Finite Element Method in Engeneering Science, McGraw-Hill, London, (2002)

2. L. Segerlind, Applied Finite Element Analysis, $2^{\text {nd }}$ edition, Wiley, New York, (1984)

3. VUZ Plc., Test report Strength tests of bogies and their components: Non-destructive testing Magnetic particle test (MT) - Bogie frame Y25Ls-K, VUZ Plc., Czech Republic, (2017)

4. EN 13749:2011 Railway applications. Wheelsets and bogies. Method of specifying the structural requirements of bogie frames, (2011)

5. EN 12663-2:2010 Railway applications. Structural requirements of railway vehicle bodies. Freight wagons, (2010)

6. Technical specifications for Interoperability (TSI) Rolling stock: Freight wagons. European Union, Agency for Railways, (2013)

7. UIC Leaflet 615-4, Motive Power Units - Bogies And Running Gear - Bogie Frame Structure Strength Tests, $2^{\text {nd }}$ edition, UIC, (2003)

8. UIC Leaflet UIC 510-3, Wagons - Strength Testing Of 2 And 3-axle Bogies On Test Rig, UIC, (1989)

9. R. Tenchev, User guide for COSMOS/M, Technical University - Sofia, (2010)

10. K. Velkov, O. Krastev, Technologies and systems for train control, Technical University - Sofia, (2010) 